\title{
Estado de conservación de los peces de la familia Goodeidae (Cyprinodontiformes) en la mesa central de México
}

\author{
Marina Y. De la Vega-Salazar \\ Departamento de Ecología Evolutiva, Instituto de Ecología UNAM. Dirección actual: Universidad de Guadalajara, Centro \\ Universitario Los Lagos. Enrique Díaz de León s/n, Colonia Paseos de la Montaña Lagos de Moreno, Jalisco, México; \\ marina_vesa@yahoo.com
}

Recibido 10-VI-2005. Corregido 11-VIII-2005. Aceptado 15-X-2005.

\begin{abstract}
Conservation status of Goodeidae familiy fishes (Cyprinodontiformes) from the Mexican Central Plateau. To establish the conservation status and threats for Goodeidae fishes in the high plateau of Mexico, I assessed limnological descriptions, and the reduction in range and in number of localities where they are found, in 53 localities ( $58.8 \%$ of historically reported localities). This assessment included the comparison of current collections with historical records. A principal component analysis of limnological variables showed that most remnant Goodeid species inhabit localities characterised by low environmental degradation: few appear to have a high tolerance to environmental degradation. Overall, $65 \%$ of species suffered a reduction in number of localities where they are found. Almost all species face some conservation threat, considering the criteria and categories established by the International Union for the Conservation of Nature (IUCN). Data suggest that one species is extinct (Allotoca catarinae), one more is extinct in the wild (Skiffia francesae), eight are critically endangered (Allodontichthys hubbsi, Allotoca goslinei, Allotoca regalis, Allotoca zacapuensi, Ameca splendens, Characodon audax, Hubbsina turneri, and Zoogoneticus tequila), eleven are endangered, eight can be regarded as vulnerable, four are "near threatened" and only two appear to under "least concern". Habitat loss, introduction of exotic fish, and being species with restricted physiographic range or ecologically specialised, are the main factors leading to the threat of extinction of the Goodeidae family. Recovery actions are needed for the conservation of this fish group. Rev. Biol. Trop. 54(1): 163-177. Epub 2006 Mar 31.
\end{abstract}

Key words: Goodeidae, Mexican endemic fish, threat of extinction, conservation.

México cuenta con cerca del $10 \%$ de todos los organismos de la tierra (Rzedowski 1991, Mittermeier y Mittermeier 1992). Su riqueza biológica radica en su diversidad, y en el elevado número de especies endémicas del país (Mittermeier y Mittermeier 1992, Ceballos 1993).

La ictiofauna dulceacuícolas en México es excepcionalmente rica, ya que tiene alrededor de 506 especies de peces distribuidas en 47 familias. Esto representa $6 \%$ de las especies conocidas en el planeta. Una breve comparación señala que Estados Unidos y Canadá, siendo 10 veces más grandes que México, cuentan con 792 especies de peces (Lydeard y Mayden 1995), lo que habla de la importancia de la ictiofauna de México, debido a la riqueza de especies, pero principalmente por que presenta altos niveles de endemismos, ya que 163 especies (32\%) son endémicas de México (CONABIO 2000).

La Mesa Central de México alberga 11 familias. Por otra parte la familia Goodeidae es la más rica en endemismos, con 36 especies que representan el $32 \%$ de las especies endémicas de la Mesa Central de México. Este sitio se ha caracterizado por una gran explotación de recursos naturales, situación 
que ha sido favorecida por los grandes asentamientos urbanos, constituyendo a esta región en una de las más densamente pobladas de la República Mexicana (Fig. 1), y también la más contaminada por actividades agrícolas, pecuarias e industriales de gran importancia económica. Todo esto ha contribuido a que los cuerpos de agua de las cuencas del LermaChapala-Santiago, Balsas, figuren como los más contaminados del país (Vázquez Gutiérrez 1993), lo que ha provocado la desaparición de la fauna y flora de sus aguas en algunas de sus porciones (Miller et al. 1989, Díaz Prado et al. 1993, Soto-Galera et al. 1999, López-López et al. 2001, De la Vega-Salazar et al. 2003 a), y donde se distribuyen más del $75 \%$ de las especies de Goodeidae, taxones, que además presentan características fisiológicas que posiblemente pueden poner en peligro su supervivencia por bioacumulación de contaminantes (De la Vega-Salazar et al. 1997).

Esta situación se refleja por el hecho de que en los 1960s solo cuatro especies fueron reportadas como extinciones recientes y 36 especies estaban claramente en peligro.
En 1979 la lista de The American Fisheries Society's indicaba 67 especies de peces en peligro y amenazadas; una década después, este número había aumentado a 123 especies, lo que representa un aumento del $83 \%$; el número reportado hasta 1993 excedía de 135 especies amenazadas o en peligro y al menos 16 especies extintas. Estos números pueden incrementarse ya que muchas especies no han sido estudiadas en cuanto a su estado de conservación (De la Vega-Salazar 2003).

La distribución histórica de los peces de la familia Goodeidae ha sido ampliamente estudiada (Bean 1899, Meek 1902, Meek 1904, Hubbs 1924, Hubbs and Turner 1939, De Buen 1941, Turner 1946, Alvarez y Cortés 1962, Kingston 1978, Radda 1984, Lyons et al. 1995, Webb and Miller 1998, Museo de la Universidad de Michigan), por lo que se conoce su área de distribución y un gran número de localidades históricas, donde han sido reportados. Sin embargo el estado de conservación actual de los Goodeidos se desconoce, por lo que es importante determinar el efecto del deterioro ambiental que enfrenta la zona central de

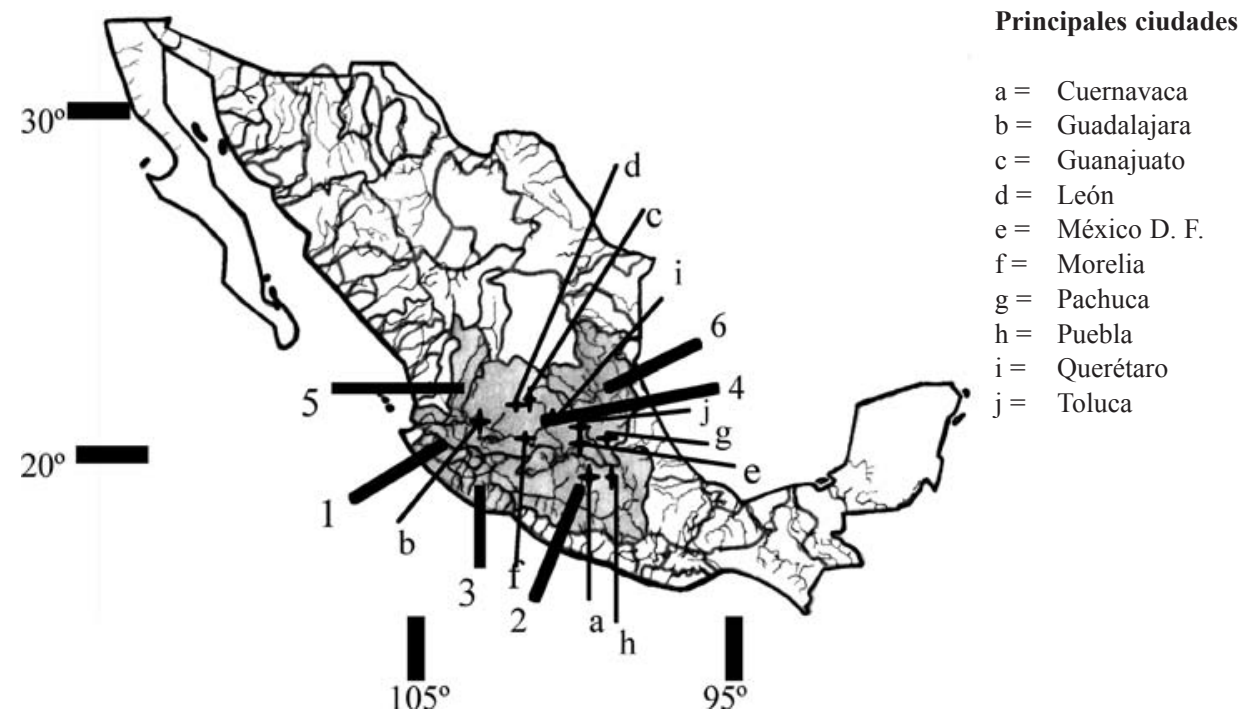

Fig. 1. Area de distribución de los peces de la familia Goodeinae (área sombreada), que incluye las principales cuencas de la zona central de México: 1. Ameca, 2. Balsas, 3. Coahuayana, 4. Lerma-Chapala-Santiago, 5. Mezquital, 6. Pánuco.

Fig. 1. Distributiion area of the Goodeinae family (dark area), and principal pleateau in the Central zone in Mexico 1. Ameca, 2. Balsas, 3. Coahuayana, 4. Lerma-Chapala-Santiago, 5. Mezquital, 6. Pánuco. 
México en la conservación de especies endémicas de México. El presente estudio se llevó a cabo para conocer el estado de conservación de los peces de la familia Goodeidae con énfasis en la zona de la Mesa Central, e identificar los factores de riesgo que enfrentan, evaluando la disminución en el número de localidades y el área de distribución, e incorporando una descripción limnológica en 53 localidades. Esta evaluación incluyó una comparación de las colecciones actuales con registros históricos de la distribución de especies de esta familia.

\section{MÉTODOS}

El género Goodea no fue incluido en esta revisión, ya que existe un gran número de localidades reportadas (alrededor de 100), además es posible encontrar peces de este género casi en cualquier cuerpo de agua dentro de su área de distribución, donde generalmente es muy abundante, por lo que no hay duda de que esta especie es muy tolerante a la degradación ambiental y no enfrenta ningún riesgo. Por tanto, en este estudio se enfocaron los esfuerzos en conocer la situación del resto de las especies de la familia.

Estado de conservación: Con base en los registros históricos de las localidades de poblaciones de Goodeidae, se seleccionaron 53 localidades (Cuadro 1), lo que corresponde al $58.8 \%$ de los sitios reportados. Se analizaron al menos el $45 \%$ de las localidades conocidas para cada especie (Cuadro 2), considerando que al menos en este sitio estuviera reportada más de una especie o que se sabía presentaba algún grado de deterioro ambiental, y revisando las localidades mínimas para cubrir el área de distribución de cada especie. En el caso de las especies con un área de distribución reducida o pocas localidades, se revisaron todas las localidades. Cuando no se encontró a la especie reportada se revisaron todas las localidades, para incrementar la certeza de ausencia de la especie en todo su ámbito.
Como parte del estudio limnológico se analizaron 53 localidades (Cuadro 1), 11 al menos ocho veces en un año (julio del 2000 a agosto del 2001): (De la Vega-Salazar et al. 2003 a). Una localidad fue estudiada en dos estaciones en 1996 (De la Vega-Salazar et al. 1997), nueve localidades se revisaron una vez en la primavera del 2000. En las restantes localidades se realizó un muestreo puntual en el otoño-invierno del 2001 (Cuadro 1).

En las localidades estudiadas se determinó la presencia de las especies, recolectando ejemplares mediante el arrastre con un chinchorro de 5.5 -m longitud $(0.4 \mathrm{~cm}$. luz de malla), y mediante el uso de trampas para peces. Únicamente se determinó presencia - ausencia de las especies estudiadas. A partir de esta muestra se determinó la proporción en la reducción del número de localidades para las especies. Para determinar el área de distribución de las especies además de las 53 localidades, se registró la presencia en 20 localidades nuevas. Usando los criterios y categorías de riesgo establecidos por la IUCN (2001), se catalogó a las especies estudiadas en las diferentes categorías de riesgo.

Estudio limnológico: Para caracterizar el hábitat se midió la velocidad de flujo con un medidor de corriente. La concentración de clorofila a, una aproximación de la biomasa fitoplanctónica y productividad, se determinó usando el método colorimétrico de Quarmby and Allen (1989). La calidad del agua se estableció midiendo el oxígeno disuelto en la superficie y fondo del cuerpo de agua. Además fueron analizados la transparencia, turbidez, $\mathrm{pH}$, así como la concentración de amonio, nitratos, nitritos y la concentración de fosfatos totales.

Clasificación de especies por su tolerancia a la degradación ambiental: Con las variables limnológicas de las localidades estudiadas se aplicó un análisis de componentes principales y al análisis de la varianza (Statistica 2000) con el fin de agrupar a las localidades estudiadas por su nivel de degradación ambiental y evaluar la asociación entre las 
CUADRO 1

Lista de localidades estudiadas

TABLE 1

Localities studied

\section{LOCALIDAD}

1 Alvaro Obregón Michoacán ${ }^{1}$

2 Amado Nervo Durango.

3 Ameca Jalisco.

4 Anteojitos San Luis Potosí

5 Ayuquila Jalisco

6 Camécuaro Michoacán

7 Chapala Jalisco ${ }^{3}$

8 Chapultepec D. F.

9 Chilchota Michoacán

10 Cobano Colima

11 Coíntzio Michoacán ${ }^{1}$

12 Colima Colima

13 Comala Colima

14 Cuitzeo Michoacán ${ }^{3}$

15 El Seco Colima

16 El Toboso Durango.

17 Hurtado Jalisco

18 I. Ramírez México ${ }^{2}$

19 Jaripo Michoacán

20 Laja Guanajuato ${ }^{1}$

21 La Maiza, Michoacán

22 Magdalena Jalisco ${ }^{3}$

23 Mintzita Michoacán ${ }^{1}$

24 Molino de Chapultepec Michoacán ${ }^{1}$

25 Opopeo. Michoacán

26 Orandino Michoacán ${ }^{1}$

27 Pátzcuaro Michoacán ${ }^{3}$

28 Pihuamo Colima

29 Potrero Grande

30 Pte. Dávalos Jalisco

31 Pte. Matlala Puebla

32 Purificación Jalisco

33 Rio Grande Morelia Michoacán ${ }^{3}$

34 Salado Jalisco.

35 San. Francisco Rincón Guanajuato ${ }^{1}$

36 San Juanico México

TIPO DE HABIT
Canal de Riego
Río
Río
Manantial
Río
Manantial- Río
Laguna
Lago
Manantial
Río
Presa-Laguna
Río
Río
Laguna
Río
Manantial
Presa
Presa
Laguna
Río
Manantial
Laguna

Manantial-Laguna

Manantial

Manantial

Manatial-Presa

Laguna

Río

Río

Río

Río

Río

Río

Río

Manantial

Presa
* PRESENTES

$2,3,35,25$

18

14

$12,22,24$

28,35

15

20

28,35

12,24

25

$21,27,35$

$12,22,24$

$12,22,24$

13,32

$3,21,25$

22, 24

17

$15,28,31,35$

19

13, 15, 35

32

27

3

5

30

$13,27,32,35$

21,25

2, 27, 32

6

$13,26,28,35$

13,32

2, 3, 27

22

11

10,22

10,22

23

22,33

21, 25, 28

12, 24,

22 
CUADRO 1

Lista de localidades estudiadas (continuación)

TABLE 1

Localities studied (continued)

\section{LOCALIDAD}

37 San Sebastián Jalisco. ${ }^{3}$

38 Sta. Catarina Michoacan

39 Santiago (Ocotlán) Jalisco ${ }^{3}$

40 Tamazula. Pte. Contla Jalisco.

41 Teuchitlán Jalisco ${ }^{1 \mathrm{p}}$

42 Teuchitlan tequila Jalisco ${ }^{1}$

43 Tierra Quemada San Luis Potosí.

44 Tocumbo Michoacán

45 Tula

46 Turbio (Manuel Doblado) Guanajuato ${ }^{3}$

47 Tuxcacuesco Jalisco

48 Tuxpan Jalisco.

4927 de Noviembre Durango.

50 Xochimilco D. F.

51 Yuriria Guanajuato ${ }^{3}$

52 Zacapu Michoacán ${ }^{1}$

53 Zirahuén Michoacán ${ }^{1}$

$\begin{array}{ccc}\text { TIPO DE HABITAT } & * \text { PRESENTES } & * \text { AUSENTES } \\ \text { Presa } & 2,5,30 & 31 \\ \text { Presa } & & 1 \\ \text { Río } & 10,12,22,24 & 11,30,31 \\ \text { Río } & 8,35 & 26,34 \\ \text { Manantial } & 34 & \\ \text { Manantial } & 29 & \\ \text { Río } & 16 & 6,13,23 \\ \text { Manantial } & & 20 \\ \text { Río } & & 13,25,32 \\ \text { Río } & 24 & 22 \\ \text { Río } & 12,22 & 9,11,30 \\ \text { Río } & 18 & 2,3,13 \\ \text { Río } & 20 & 3,21 \\ \text { Canales } & 13,32 & 28 \\ \text { Laguna } & 7,13,28 & \\ \text { Manantial-Laguna } & & \\ \text { Laguna } & & \end{array}$

Incluyendo once localidades estudiadas de julio del 2000 a agosto del 20011 (De La Vega-Salazar et al. 2003 a). Una localidad fue estudiada en dos estaciones en 1996 (De la Vega- Salazar et al. 1997)2. Diez localidades se revisaron una vez en el 2000 3, el resto de localidades se estudiaron una vez en otoño - invierno 2001. * Especies de Goodeidos históricamente reportadas, para el número de especie ver cuadro 2.

características de las diferentes localidades y la presencia o ausencia de las especies reportadas históricamente.

Se catalogaron a las especies según su tolerancia a la degradación ambiental: 1) Las especies sensibles a la degradación ambiental: Son las especies que sólo ocurren en localidades sin degradación ambiental y fueron desplazadas de localidades con algún grado de degradación ambiental. 2) Las especies con tolerancia media a la degradación ambiental: Son las especies que no están restringidas a localidades sin degradación ambiental, pero fueron desplazadas de localidades con mayor degradación ambiental. 3) Las especies tolerantes a la degradación ambiental: Son especies que inclusive se encuentran en localidades con mayor degradación ambiental.

\section{RESULTADOS}

Algunas especies se encontraron en localidades no registradas históricamente, como parte de otro estudio (De la Vega Salazar et al. 2003 a y b) (Cuadro 2). Estos datos no se consideran en el cálculo de la reducción del número de localidades ya que se desconoce si son nuevas localidades o si sólo son sitios que no se habían estudiado anteriormente, y porque pudieran encubrir el resultado de la reducción en el número de localidades históricas, sólo se 
CUADRO 2

Número de localidades (Loc.) reportadas y estudiadas para cada especie

TABLE 2

Number of reported and studied localities for each species

\section{ESPECIE}

Allotoca catarinae. De Buen 1942

Allotoca diazi. Meek 1902

Allotoca dugesi. Bean 1887

Allotoca goslinei. Smith y Miller 1987

Allotoca maculata. Smith y Miller 1980

6 Allotoca regalis. Alvarez 1959

7

Allotoca zacapuensis. Meyer, Rada y

Domínguez 2001

8 Ameca splendens. Miller y Fitzsimons 1971

9 Allodontichtys hubbsi. Miller y Uyeno 1980

10 Allodontichtys polylepis. Rauchenberger 1988

11 Allodontichtys tamazulae. Turner 1946

12 Allodontichtys zonistius. Hubbs 1932

13 Alloophorus robustus. Bean 1892

14 Ataeniobius toweri. Meek 1904

15 Chapalichthys encaustus. Jordan y Snyder 1900

16 Chapalichthys pardalis. Alvarez 1963

17 Characodon audax. Jordan y Everman 1898

18 Characodon lateralis. Günther 1866

19 Girardinichthys multiradiatus. Meek 1904

20 Girardinichthys viviparus. Bustamante 1837

21 Hubbsina turneri. De Buen 1941

22 Ilyodon furcidens. Jordan y Gilbert 1882

23 Ilyodon whitei. Meek 1904

24 Ilyodon xantusi. Hubbs y Turner 1939

25 Skiffia bilineata. Bean 1887

26 Skiffia francesae. Kingston 1978

27 Skiffia lermae. Meek 1902

28 Skiffia multipunctata. Pellegrin 1901

29 Xenoophorus captivus. Hubbs 1924

30 Xenotoca eiseni. Rutter 1896

31 Xenotoca melanosoma. Fitzimons 1972

32 Xenotoca variata. Bean 1887

33 Xenotaenia resolanae. Turner 1946

34 Zoogoneticus tequila. Webb y Miller 1998

35 Zoogoneticus quitzeoensis. Bean 1898
$\mathrm{N}^{\circ}$ de registro colección MZUM

242417

233518

189047

212324

173553

202428

*25811

202412

172153

198850

202621

230452

233554

196341

192528

179226

213312

166708

192376

189664

233565

230436

181313

160762

189034

172223

192419

201582

189284

173555

212322

190896

160927

233655

233529
$\mathrm{N}^{\circ} \mathrm{de}$

Loc. Loc. Reportadas Estudiadas

\%de Loc.

$\mathrm{N}^{\circ}$ de

Estudiadas Loc.

nuevas

$\begin{array}{llll}1 & 1 & 100 & 0\end{array}$

$\begin{array}{llll}5 & 3 & 60 & 2\end{array}$

$\begin{array}{lll}9 & 5 & 55\end{array}$

100

100

100

100

100

100

100

100

67

62

75

66

60

50

100

100

50

62

100

62

25

75

100

100

100

88

50

50

50

55

50

100

65
0

$$
1
$$$$
0
$$

0

1

$$
0
$$$$
0
$$$$
0
$$$$
1
$$$$
0
$$

0

3

0

2

$$
1
$$$$
0
$$$$
0
$$

$$
3
$$

0

0

$$
6
$$$$
0
$$

2

1

0

0

$$
1
$$$$
1
$$$$
2
$$

7

6

0

0

3

MZUM = Museo de Zoología. Universidad de Michigan, *= Museo de Historia Natural de Viena. 
consideran para determinar el área de distribución actual de cada especie.

Considerando los registros históricos, hay especies con amplia distribución geográfica, con distribución media, y con distribución reducida, en los tres grupos se presenta la reducción en el número de localidades y área de distribución (Fig. 2, Cuadro 3). El 69.5\% (25) de las especies presentaron una disminución en el número de localidades. Seis especies (16.7\%) presentan el $100 \%$ de reducción en el número de localidades, tres especies $(8.3 \%)$ más del $75 \%$ de reducción en el número de localidades y ocho especies $(22.2 \%)$ más del $50 \%$ en la reducción del

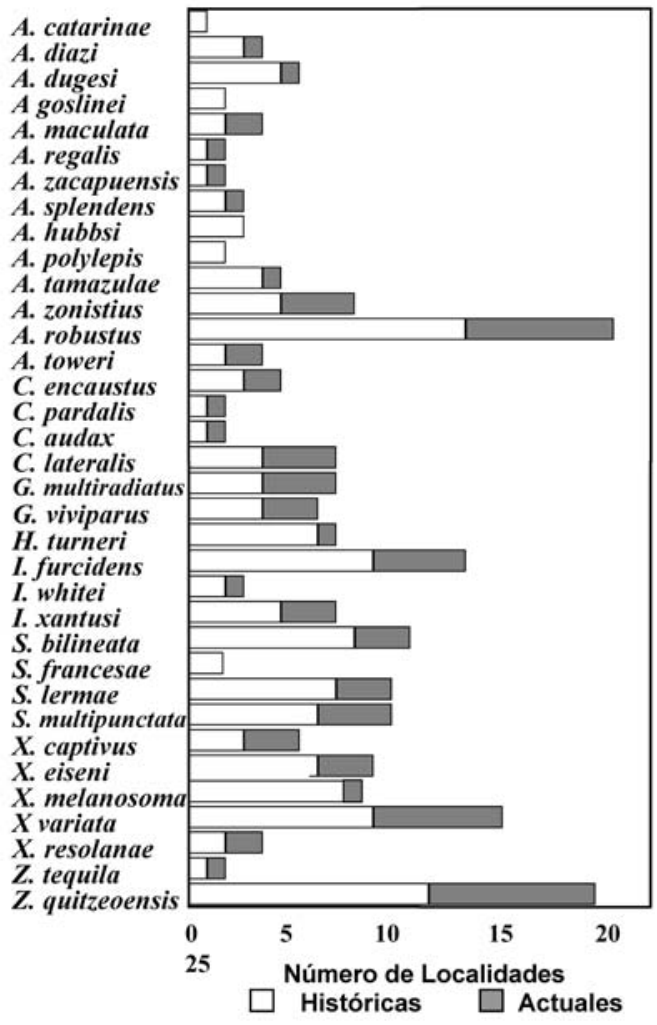

Fig. 2. Relación entre el número de localidades estudiadas donde, fueron históricamente reportadas especies de goodeidos y el número de localidades donde actualmete es posible encontras a las especies estudiadas.

Fig. 2. Relationship between the number of historical localities where species of goodeids were reported, and the number of localities where is possible to find the studied species currently. número de localidades (Cuadro 3, Fig. 3). Solo ocho especies $(24.4 \%)$ no presentan reducción en el número de localidades.

La disminución en el número de localidades ha llevado a una reducción en el área de distribución a 18 especies $(50 \%)$. Sobresalen cuatro especies con el $100 \%$ de reducción: A. catarinae, A. goslinei, A. hubbsi, S. francesae. H. turneri con el $99.8 \%$ de reducción, $Z$. tequila con el $99.5 \%$ de reducción, A. dugesi, con el $99 . \%$ de reducción $X$. melanosoma y $A$. splendens con el $90 \%$, S. lermae $86 \%$, S. bilineata $80 \%$ y $X$. eiseni $75 \%$ (Cuadro 3).

En nueve de las localidades estudiadas no se encontró ninguna de las especies reportadas históricamente, y en 19 localidades fue desplazada al menos una especie (Cuadro 1) lo que indica que el $53 \%$ de las localidades no presentan condiciones óptimas para mantener poblaciones de peces de la familia.

El estudio limnológico mostró que gran número de localidades presentan algún grado de deterioro ambiental, 13 localidades presentan un grado severo de eutrofización y son las localidades de donde se han desplazado el mayor número de especies, 11 localidades presentan un grado de eutrofización intermedio y en la mayoría de ellas se han desplazado especies (Cuadro 1, Fig. 4). Hay localidades como Zirahuén que aunque no presenta algún grado de deterioro ambiental, si presenta la

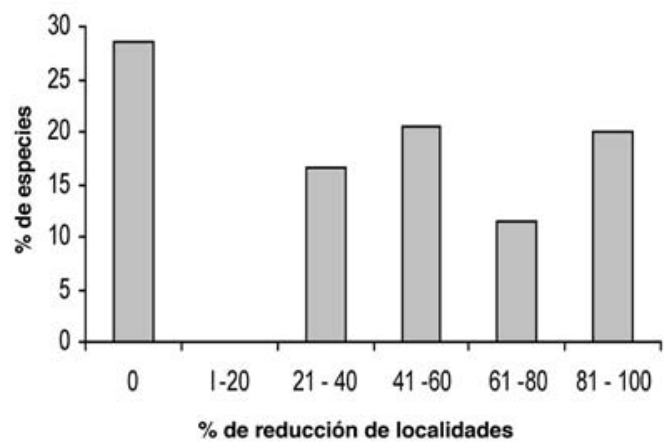

Fig. 3. Frecuencia de la proporción de reducción de localidades estudiadas para las especies de Goodeidos.

Fig. 3. Reduction of localities frequencies in Goodeids species. 
CUADRO 3

Proporción de la reducción de localidades y área de distribución de las especies estudiadas

TABLE 3

Proportion of reduction in localities and distribution area of studied species

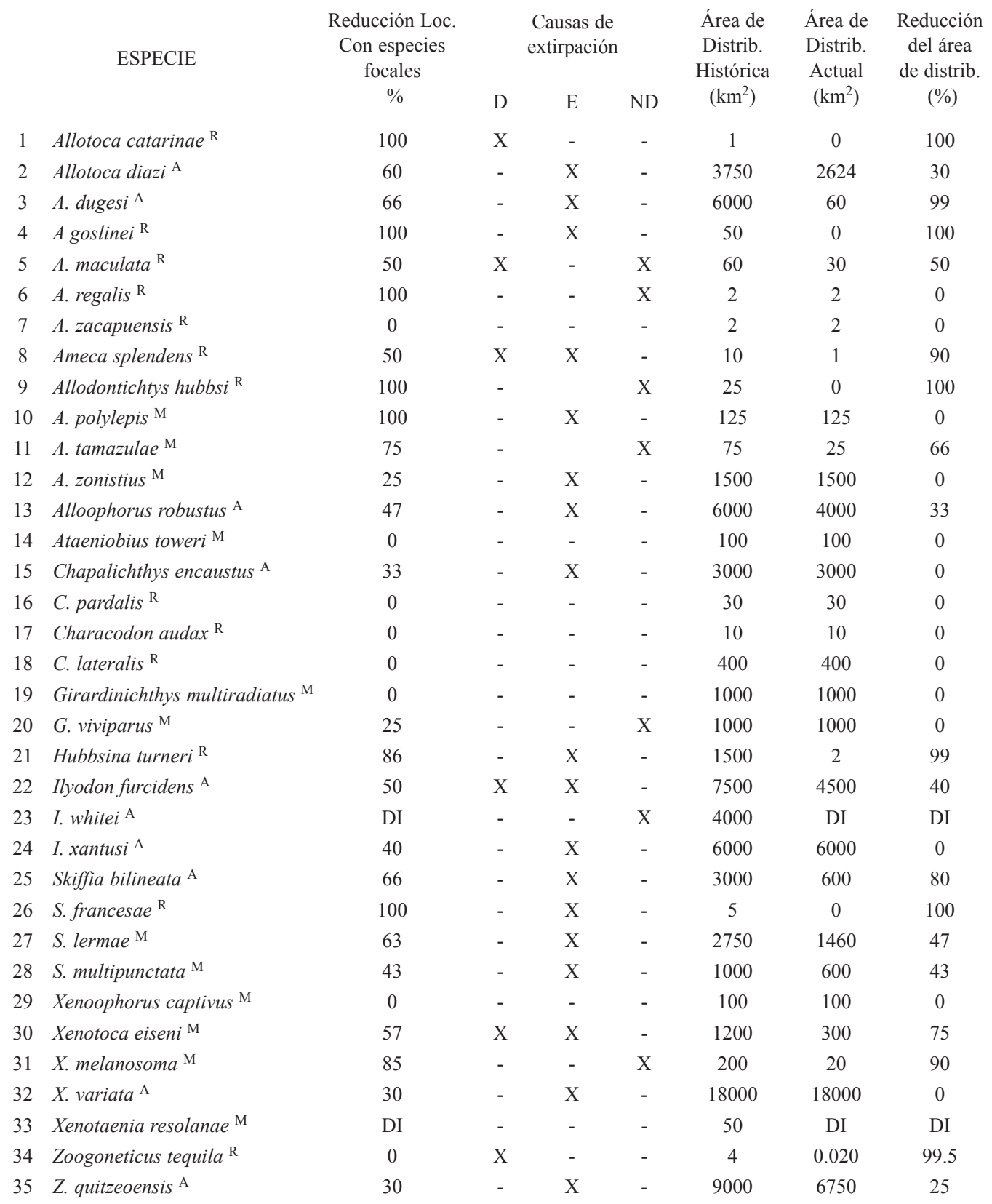

Área de distribución de la especie: $\mathrm{A}=$ Amplia. $\mathrm{M}=$ Media, $\mathrm{R}=$ Reducida. $\mathrm{DI}=$ Datos insuficientes Causas de extirpación de especies por localidad. $\mathrm{D}=$ Desaparición o fragmentación de hábitat. $\mathrm{E}$ = Eutroficación y contaminación de hábitat, $\mathrm{ND}=$ No determinado. 
introducción de la especie exótica carnívora Mycropterus salmoide.

En el análisis de componentes principales de las variables limnológicas evaluadas, los dos primeros componentes principales explican el $47 \%$ de la varianza. El primer componente esta asociado principalmente a la concentración de oxígeno, transparencia y turbidez, el segundo esta asociado a velocidad de flujo y concentración de clorofila (productividad). La concentración de oxígeno disuelto fue mayor en localidades con goodeidos $(9.28 \mathrm{ppm} \pm$ 4.51) que sin goodeidos (4.09 ppm \pm 4.46 ), lo mismo que la velocidad de flujo, mientras que la turbidez, concentraciones de amonio fosfatos totales y clorofila fueron mucho menores en las localidades con goodeidos (Cuadro 4).

Los resultados sugieren que las localidades sin goodeidos presentan características muy diferentes a las localidades con goodeidos, sin embargo podemos considerar un grupo intermedio de localidades donde persisten algunas especies y otras han sido desplazadas. Por lo que podemos distinguir tres grupos de localidades (Fig. 4). Las localidades con concentraciones de oxígeno disuelto altas, aguas muy transparentes y niveles bajos de eutrofización (I), localidades con altos niveles de eutrofización, concentraciones bajas de oxígeno disuelto $\mathrm{y}$ altos niveles de turbidez (III), y un tercer grupo (II) con sitios que presentan un grado intermedio entre las características mencionadas (Fig. 4).

De acuerdo con el resultado de componentes principales de los tres grupos de localidades se realizó la comparación de las principales variables limnológicas usando el análisis de varianza. El análisis de varianza por grupo

\section{CUADRO 4}

Analisis de varianza de las variables limnológicas por grupos de localidades, (ver fig. 4), donde se encontraron diferencias significativas $(p<0.05)$

TABLE 4

ANOVA of limnoligical variables by group of localities, (see fig. 4), where significant differences ( $p<0.05$ ) were found

\begin{tabular}{|c|c|c|c|c|c|c|}
\hline $\begin{array}{c}\text { GRUPO DE } \\
\text { LOCALIDADES }\end{array}$ & $\begin{array}{c}\text { Turbidez } \\
\text { UFT }\end{array}$ & $\begin{array}{l}\text { Oxígeno } \\
\text { PPM }\end{array}$ & $\begin{array}{l}\mathrm{NH}_{4} \\
\mathrm{PPM}\end{array}$ & $\begin{array}{l}\mathrm{PO}_{4} \\
\mathrm{PPM}\end{array}$ & $\begin{array}{l}\text { Clorofila } \\
\text { PPM }\end{array}$ & $\begin{array}{c}\text { Vel. De Flujo } \\
\mathrm{cm} / \mathrm{seg}\end{array}$ \\
\hline I & $\begin{array}{c}x=3.09 \\
\sigma=1.9\end{array}$ & $\begin{array}{c}x=12.46 \\
\sigma=4.21\end{array}$ & $\begin{array}{l}x=0.06 \\
\sigma=0.09\end{array}$ & $\begin{array}{c}x=0.94 \\
\sigma=2.2\end{array}$ & $\begin{array}{l}x=0.039 \\
\sigma=0.017\end{array}$ & $\begin{array}{l}x=19.0 \\
\sigma=18.3\end{array}$ \\
\hline II & $\begin{array}{c}x=19.96 \\
\sigma=6.17\end{array}$ & $\begin{array}{l}x=6.09 \\
\sigma=1.65\end{array}$ & $\begin{array}{l}x=0.47 \\
\sigma=0.38\end{array}$ & $\begin{array}{c}x=1.63 \\
\sigma=2.4\end{array}$ & $\begin{array}{l}x=0.16 \\
\sigma=0.11\end{array}$ & $\begin{array}{l}x=5.2 \\
\sigma=7.9\end{array}$ \\
\hline III & $\begin{array}{c}x=303.09 \\
\sigma=191.3\end{array}$ & $\begin{array}{l}x=4.09 \\
\sigma=4.46\end{array}$ & $\begin{array}{c}x=6.21 \\
\sigma=4.7\end{array}$ & $\begin{array}{l}x=2.7 \\
\sigma=2.8\end{array}$ & $\begin{array}{l}x=0.34 \\
\sigma=0.17\end{array}$ & $\begin{array}{l}x=0.20 \\
\sigma=0.63\end{array}$ \\
\hline I-II & $\begin{array}{c}F=3.23 \\
p=0.002\end{array}$ & $\begin{array}{l}\mathrm{F}=13.17 \\
\mathrm{p}=0.001\end{array}$ & $\begin{array}{l}F=1.06 \\
p=0.33\end{array}$ & $\begin{array}{l}F=1.48 \\
p=0.17\end{array}$ & $\begin{array}{c}F=5.3 \\
p=0.006\end{array}$ & $\begin{array}{l}F=2.65 \\
p=0.71\end{array}$ \\
\hline II-III & $\begin{array}{l}\mathrm{F}=12.52 \\
\mathrm{p}=0.001\end{array}$ & $\begin{array}{l}\mathrm{F}=2.31 \\
\mathrm{p}=0.06\end{array}$ & $\begin{array}{r}\mathrm{F}=3.23 \\
\mathrm{p}=0.003\end{array}$ & $\begin{array}{l}F=2.12 \\
p=0.62\end{array}$ & $\begin{array}{l}F=4.03 \\
p=0.011\end{array}$ & $\begin{array}{l}F=3.78 \\
p=0.032\end{array}$ \\
\hline III-I & $\begin{array}{l}F=5.62 \\
p=0.001\end{array}$ & $\begin{array}{l}F=10.62 \\
p=0.0001\end{array}$ & $\begin{array}{l}F=10.43 \\
p=0.0001\end{array}$ & $\begin{array}{l}F=3.03 \\
p=0.014\end{array}$ & $\begin{array}{c}F=8.4 \\
p=0.001\end{array}$ & $\begin{array}{l}F=4.52 \\
p=0.002\end{array}$ \\
\hline
\end{tabular}

$\mathrm{x}=$ promedio, $\sigma=$ desviación estándar. 


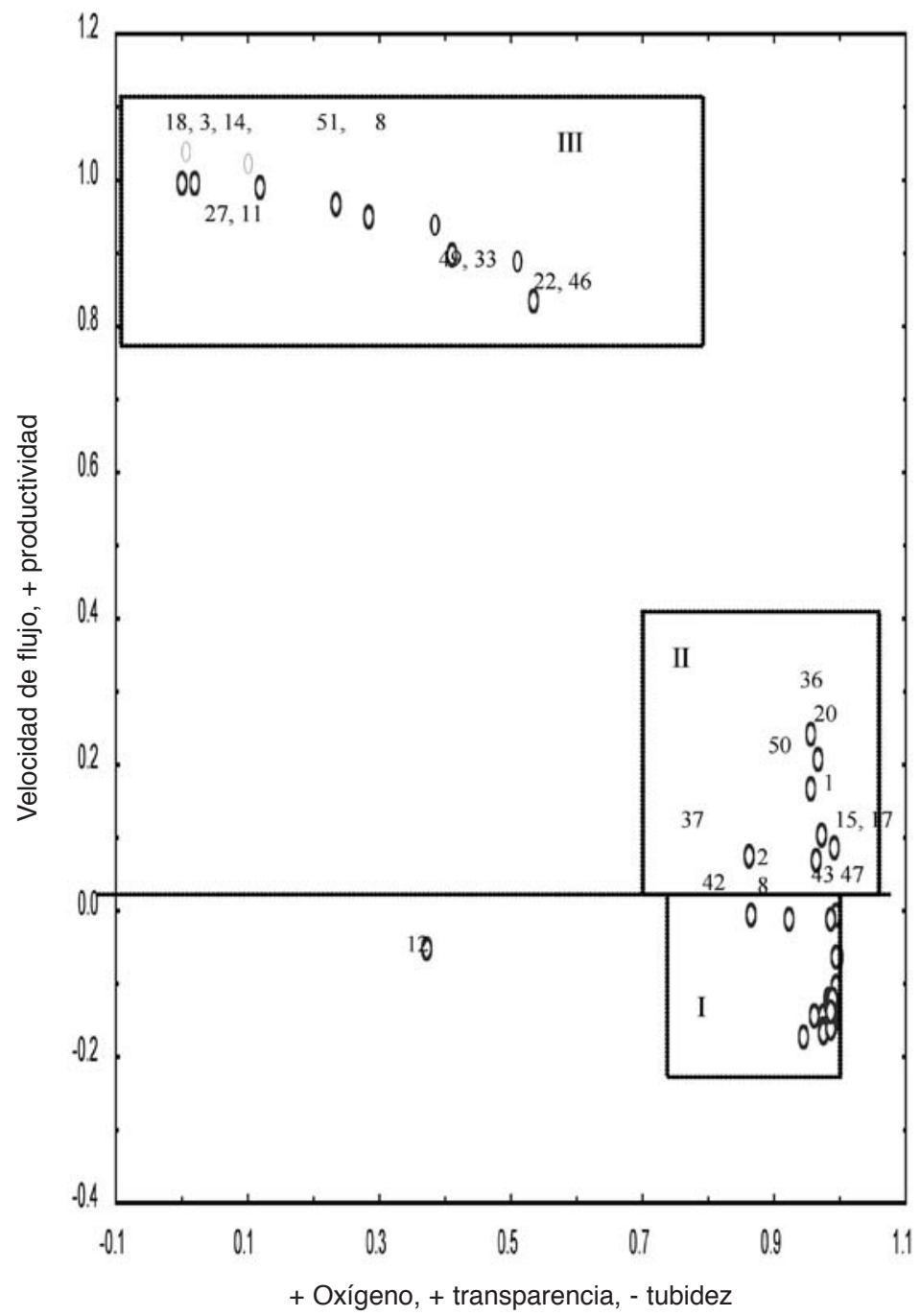

Fig. 4. Análisis de componentes principales de las variables fisicoquímicas evaluadas en las localidades estudiadas. (Los números indican las localidades, ver Cuadro 1).

Fig. 4. Principal components analysis of physicochemical variables in localities studied, (Number indicates localities, see Cuadro 1).

de localidades mostró que hay diferencias significativas $(\mathrm{p}<0.05)$ para la transparencia, oxígeno disuelto, velocidad de flujo, NH4, PO4 y Clorofila (Cuadro 4).

Con base en el análisis de componentes principales de las variables limnológicas y la distribución de especies por localidad (Fig. 4, Cuadro 1) y considerando las características de las localidades donde fueron desplazadas o subsisten las especies, se pueden clasificar a las especies de goodeidos en tres grupos: Especies sensibles a la degradación ambiental, incluyendo a: A. catarina, A. goslinei, A. regalis, A. splendens, A. tamazulae, A. zonistius, $H$. turneri, I. fursidens, I. xantusi, S. francesae y S. lermae. Las especies con tolerancia media a la degradación ambiental: A. diasi, A. dugesi, A. polilepis, C. pardalis, S. multipunctata, 
X. captivus, Z. tequila, Z. quitzeoensis. Las especies tolerantes a la degradación ambiental: A. maculata, A. robustus, C. encaustus, G multirradiatus, G. viviparus, $S$. bilineata, X. eiseni, $X$. melanosoma y $X$. variata.

Algunas de las especies que tienen mayor tolerancia a la degradación ambiental son las especies que tienen mayor área de distribución, sin embargo varias de estas especies también han sido desplazadas de algunas localidades principalmente por degradación ambiental (Cuadro 3).

\section{DISCUSIÓN}

En las localidades donde no se encontraron las especies reportadas (Cuadro 1), la ausencia es reforzada por criterios como son: sitios que no tienen condiciones para mantener peces como son las localidades sin agua o sin oxígeno; que reciben principalmente descargas de aguas residuales; o que se ha reportado su ausencia en otros trabajos recientemente publicados (LópezLópez et al. 1991, Díaz-Prado et al. 1993, SotoGalera et al. 1999, López-López et al. 2001, De la Vega-Salazar et al. 2003 a y b).

En el caso de las especies desplazadas de todas sus localidades históricas, no se realizó una búsqueda exhaustiva, con excepción de $S$. francesae y Z. tequila (De la Vega-Salazar et al. 2003 a y b). En el caso de A. catarinae no se considera la posibilidad de encontrarla en otras localidades ya que las características fisiográficas y la degradación ambiental de las zonas circundantes son barreras importantes para pensar que la especie encontrara refugio en zonas cercanas

Las especies de goodeidos persisten principalmente en localidades con altas concentraciones de oxígeno y aguas más transparentes, y de preferencia con corriente, y bajas concentraciones de amonio, fosfatos y sitios poco eutrofizados. Los resultados sugieren que la degradación ambiental es la principal causa de desplazamiento de especies de Goodeidos de sus localidades históricas.
Las actividades antropogénicas han ocasionado que gran número de cuerpos de agua estén eutrofizados y tengan gran acumulación de sedimentos, características que han propiciado que las especies sensibles y con tolerancia media a la degradación ambiental estén siendo desplazadas de muchas localidades, por lo que actualmente gran número de especies de Goodeidos se encuentran en riesgo, principalmente por que no existen medidas efectivas que detengan el deterioro ambiental y menos aun que lo reviertan. En toda la zona estudiada se observaron problemas de degradación ambiental sin embargo las cuencas del Lerma-Chapala-Santiago y de Ameca son las que presentan mayor degradación y donde se han desplazado gran número de especies de Goodeidos. (López-López et al. 1991, DíazPrado et al. 1993, Soto-Galera et al. 1999, López-López et al. 2001, De la Vega-Salazar et al. 2003 a).

Hay un grupo de especies A. zacapuensis, A. hubbsi, A. toweri, C. audax, C. lateralis, I. xantusi y $X$. resolanae, que sólo se encuentran en sitios oligotróficos, pero no hay evidencia de que fueran desplazadas de localidades con algún grado de degradación ambiental. Estas especies pueden estar limitadas a esas localidades por barreras geográficas, pero también pudieron no haber colonizado otras localidades por limitaciones fisiológicas.

Los resultados de la reducción en el área de distribución de cada especie y la reducción en el número de localidades donde actualmente se encuentran las especies, muestran que, éstos se pueden considerar en diferentes niveles de riesgo, de acuerdo con los criterios establecidos por la (IUCN 2001).

Los resultados sugieren que: Una especie muy probablemente está extinta, A. catarinae; una especie está extinta en la naturaleza; $S$. francesae, ya que sólo persiste en cautiverio; ocho se encuentran en la categoría de peligro crítico (Cuadro 5); once se encuentran en peligro, ocho pueden ser consideradas como vulnerables, cuatro se pueden considerar en riesgo próximo y solo dos parecen estar sin ningún 
CUADRO 5

Estado de conservación de las especies estudiadas de la familia Goodeidae, de acuerdo a los criterios y categorias de la IUCN (2001)

TABLE 5

Consevation state of studied species from Goodeidae family, considering IUCN (2001) criteria and categories

Especie Extincta Peligro critico En peligro Vulnerable $\begin{gathered}\text { Riesgo } \\ \text { proximo }\end{gathered}$ Sin riesgo

Allotoca catarinae

Allotoca diazi

A. dugesi

A goslinei

A. maculata

A. regalis

A. zacapuensis

Ameca splendens

Allodontichtys hubbsi

A. polylepis

A. tamazulae

A. zonistius

Alloophorus robustus

Ataeniobius toweri

Chapalichthys encaustus

C. pardalis

Characodon audax

C. lateralis

Girardinichthys multiradiatus

G. viviparus

Goodea sp.

Hubbsina turneri

Ilyodon furcidens

I. xantusi

Skiffia bilineata

S. francesae

S. lermae

S. multipunctata

Xenoophorus captivus

Xenotoca eiseni

$X$. melanosoma

$X$ variata

Zoogoneticus tequila

Z. quitzeoensis
X

X

X

X

X

X

X

X

$\mathrm{X}$

X

$\mathrm{X}$

X

X

X

$\mathrm{X}$

X

X

X

X

X

$x$

X

X

X

$$
\begin{aligned}
& X \\
& X \\
& X
\end{aligned}
$$

X

X

X 
riesgo. De dos especie, I. white y $X$. resolanae, no se tienen datos suficientes.

El primer paso para la conservación de las especies es identificar a los taxones que están en peligro y los factores de riesgo. La principal característica entre las especies extintas (A. catarinae y $S$. francesae) y las especies en peligro crítico (A. goslinei, A. regalis, A. zacapuensis, A. splendens, A. hubbsi, H. turneri, C. audax y $Z$. tequila) es su ámbito geográfico restringido, lo que confirma que para las especies de Goodeidos, las especies que tienen un ámbito geográfico restringido se encuentran en mayor riesgo.

Aunque la degradación ambiental es el principal factor causante del declive de estas especies, hay otros factores importantes como son: el ámbito geográfico limitado, la especialización ecológica y posiblemente otros factores inherentes a la biología de las especies, como ocurre en el caso del género Allotoca, el cual consta de siete especies, de las cuales una esta extinta y tres se encuentran en peligro crítico y dos en peligro (Cuadro 5). Otros factores se han reconocido como causantes de desplazamiento de especies, particularmente la fragmentación e introducción de especies exóticas (LópezLópez et al. 1991, Díaz-Prado et al. 1993, De la Vega-Salazar et al. 2003a), ya que se reduce el número de especies que pueden mantener los ecosistemas aumentando las presiones de selección, que pueden incrementar el riesgo para las especies estudiadas, por lo que deberán hacerse estudios más detallados e implementar programas específicos para cada especie con el fin de conservarlas.

Se pueden reconocer varios factores potenciales responsables de la desaparición de poblaciones de peces. La mayoría son el resultado de múltiples efectos acumulados, principalmente de impactos antropogénicos.

Los ecosistemas dulceacuícolas en México son específicamente vulnerables debido a la degradación y sobreexplotación a que están sometidos, lo que dificulta establecer estrategias de conservación para las especies de la familia. Sin embargo se reconoce la importancia de establecer programas específicos con el fin de proteger la calidad del agua y el hábitat, y para asegurar la conservación de la flora y fauna de aguas dulces.

\section{AGRADECIMIENTOS}

A CONACYT (proyecto 32005-N), por el apoyo económico otorgado para la realización de este trabajo. A Rodolfo Dirzo, Ignacio Méndez e Isaías Salgado por la revisión del manuscrito, a Edgar Ávila por su invaluable ayuda en el trabajo de campo y en la identificación de las especies. Este trabajo formó parte de mi tesis doctoral durante la cual fui beneficiada con becas de CONACYT y DGEP-UNAM.

\section{RESUMEN}

Se estableció el estado de conservación y se identificaron los riesgos que presentan los peces de la familia Goodeidae en la Mesa Central de México. Para ello se evaluó la disminución en el número de localidades y el área de distribución, y se incorporó una descripción limnológica en 53 localidades. Esta evaluación incluyó una comparación de las colecciones actuales con registros históricos de la distribución de la familia. Un análisis de componentes principales de las variables limnológicas indicó que la mayoría de las especies de goodeidos habitan localidades caracterizadas por tener poca degradación ambiental, y sólo pocas especies parecen tener elevada tolerancia a dicho estado de contaminación. Los resultados de la disminución histórica de localidades y del área de distribución sugieren que el estado de conservación de las especies (de acuerdo con los criterios de la IUCN) es el siguiente: una especie está extinta (Allotoca catarinae), una especie está extinta en la naturaleza (Skiffia francesae),ya que existen ejemplares en cautiverio, ocho están en peligro crítico (Allotoca goslinei, Allotoca regalis, Allotoca zacapuensis, Allodontichthys hubbsi, Ameca splendens, Characodon audax, Hubbsina turneri y Zoogoneticus tequila, once están en peligro, ocho se consideran como vulnerables, cuatro se pueden considerar en riesgo próximo y sólo dos parecen no enfrentar ningún riesgo. En conclusión, la pérdida de hábitat, la introducción de especies exóticas de peces, el ámbito geográfico restringido y la especialización ecológica de las especies son los principales factores de riesgo para las especies estudiadas de la familia Goodeidae. La supervivencia de este grupo requiere acciones para su conservación. 
Palabras clave: Goodeidae, fauna endémica mexicana, peligro de extinción, conservación.

\section{REFERENCIAS}

Alvarez, J., M. \& T. Cortés. 1962. Ictiología Michoacana I.-Claves y catálogos de las especies conocidas. Anal. Esc. Nac. Cienc. Biol. México XI: 79-142.

Bean, B.A. 1899. Notes on a collection of fishes from Mexico, with description of a new species of Platypoecilus. Proc. U. S. Nat. Muse. XXI No 1159: 539-542.

Ceballos, G. 1993. Especies en peligro de extinción. Rev. Dif. Ciencias D. F. México. No especial 7: 5-10.

CONABIO. 2000. Estrategia Nacional Sobre Biodivrsidad de México. Comisión Nacional para el Conocimiento y Uso de la Biodiversidad. D. F.México 103 p.

De Buen, F. 1941. Un nuevo género de la familia Goodeidae perteneciente a la fauna ictiológica Mexicana. Anal. Esc. Nac. Cienc. Biol. México 2: 133-140.

De Buen, F. 1947. Investigaciones sobre la ictiología mexicana. An. Inst. Biol. Mex. XVIII: 292-335.

De la Vega-Salazar, M.Y. 2003. Situación de los peces dulceacuícolas de México. Rev.Dif. Ciencias D. F. México 78: 20-30.

De la Vega-Salazar, M.Y., E Avila-Luna \& C. Macías-García 2003 a. Ecological evaluation of local extinction: The case of two genera of endemic fish, Zoogoneticus and Skiffia. Biod. \& Conserv. 12: 2043-2056.

De la Vega-Salazar. M.Y., E. Avila-Luna \& C. MacíasGarcía. 2003 b. Threatened fishes of the word: Zoogoneticus tequila. Environ. Biol. Fish. 68: 14.

De la Vega-Salazar, M.Y., L Martínez-Tabche, \& C. Macías-García. 1997. Bioaccumulation of Methyl Parathion and its Toxicology in Several Species of the Freshwater Community in Ignacio Ramirez dam in Mexico. Ecotox. Environ. Safety 38: 53-62.

Díaz-Prado, E., M.A. Godines-Rodriguez, E. López-López \& E. Soto-Galera. 1993. Ecología de los peces de la cuenca del río Lerma, México. Anal. Esc. Nac. Cienc. Biol. México 39: 103-127.

Hubbs, C.L. 1924. Studies of the fishes of the order Cyprinodontes. V. Notes on species of Goodea and Skiffia. Occ. Pap. Muse. Zool., University of Michigan 148, 8 pp.
Hubbs, C.L. \& C.L. Turner. 1939. Studies of the fishes of the order Cyprinodontes. XVI. A revision of the Goodeidae. Misc. Publ. Muse. Zool., University of Michigan 42, $80 \mathrm{p}$.

IUCN. 2001. IUCN Red List Categories and Criteria: Versión 3.1. IUCN Species Survival Commission. IUCN, Gland, Suiza. 30 p.

Kingston, D.I. 1978. Skiffia francesae, a new species of goodeid fish from Western Mexico. Copeia 1978: 503-508.

López-López, E. \& E. Díaz-Pardo. 1991. Cambios distribucionales en los peces del río de La Laja (Cuenca Río Lerma), por efecto de disturbios ecológicos. Anal. Esc. Nac. Cienc. Biol. México 35: 91-116.

López-López, E. \& J. Paulo-Maya. 2001. Changes in the fish assemblage in the upper Río Ameca México. J. Freshw. Ecol. 16: 179-187.

Lydeard, C. \& R.L. Mayden. 1995. A Diverse and Endangered Aquatic Ecosystem of the Southeast United States. Conserv. Biol. 9: 800-805.

Lyons, J.S., P. Navarro-Pérez, E. Cochran-Santana \& M. Guzmán Arroyo. 1995 Index of biotic integrity based on fish assembleges for the conservation of streams and river in West-Central Mexico. Conserv. Biol. 9: 569-584.

Meek, S.E. 1902. A contribution to the ichthyology of Mexico. Field Col. Muse. Publ. 65 Zool. Series, 3(6): 63-128.

Meek, S.E. 1904. The fresh water fishes of Mexico north of the Isthmus of Tehuantepec. Field Col. Muse. Publ. Zool. Series, V.

Miller, R.R. 1986. Composition and derivation of the freshwater fish fauna of Mexico. An. Es. Nac. Cienc. Biol. Mexico. 30: 121-153.

Miller, R.R., D. Williams \& J.E. Williams. 1989. Extinctions of North American fishes during the past century. Fisheries 14: 22-38.

Mittermeier, R.A. \& C. Mittermeier. 1992. La Importancia de la Diversidad Biológica de México. In J. Sarukkán y R. Dirzo (eds.). México Frente a los Retos de la Biodiversidad. CONABIO, México, pp. 63-73.

Quarmby, C. \& S.E. Allen. 1989. Organic Constituents. In S.E. Allen (ed.). Chemical analysis of ecological materials, pp 160-200. Blakwel Scientific, Oxford, Reino Unido. 
Radda, A.C. 1984 Synopsis der Goodeiden Mexikos. Killifische aus aller welt. Otto Hofmann. Vienna, Austria.

Rzedowski, J. 1991. Diversidad y Orígenes de la Flora Fanerogámica de México. Acta Bot. Méx. 14: 3-21.

Soto-Galera, E., J. Paulo-Maya, E. Lopez-Lopez, J.A. Serna-Hernández \& J. Lyon. 1999. Changes of fish fauna as indicator of aquatic ecosystem condition in Río Grande de Morelia-Lago de Cuitzeo basin, Mexico. Environ. Management. 24: 133-140.
Turner, C.L. 1946. A contribution to the taxonomy and zoogeography of the goodeid fishes. Occ. Pap. Muse. Zool., University of Michigan 495. 15 p.

Vázquez-Gutiérrez, F. 1993 Desarrollo industrial de las cuencas en México. Curso de Limnología Aplicada. Instituto de Ciencias del Mar y Limnología. D.F. México p. 34-39.

Webb, S.A. \& R.R. Miller. 1998. Zoogoneticus tequila a new goodeid fish (Cyprinodontiformes) from the Ameca drainage of Mexico, and rediagnosis of the genus. Occ. Pap. Museum of Zool., University of Michigan 725: 23 p. 
